\title{
A factorization formula in $\mathbb{Z}[[x]]$
}

\author{
Daniel Birmajer ${ }^{1} \quad$ Juan B. Gil ${ }^{2} \quad$ Michael D. Weiner ${ }^{2}$ \\ ${ }^{1}$ Department of Mathematics, Nazareth College, 4245 East Ave., Rochester, NY 14618 \\ ${ }^{2}$ Penn State Altoona, 3000 Ivyside Park, Altoona, PA 16601
}

\begin{abstract}
Given an odd prime $p$, we give an explicit factorization over the ring of formal power series with integer coefficients for certain reducible polynomials whose constant term is of the form $p^{w}$ with $w>1$. Our formulas are given in terms of partial Bell polynomials and rely on the inversion formula of Lagrange.

Résumé. Donné un nombre premier impair $p$, nous donnons une factorisation explicite sur l'anneau des séries formelles à coefficients entiers pour certains polynômes réductibles dont le terme constant est de la forme $p^{w}$ avec $w>1$. Nos formules sont donnés en termes de polynômes partiels de Bell et comptent sur la formule d'inversion de Lagrange.
\end{abstract}

Keywords: Factorization of formal power series, partial Bell polynomials

\section{Introduction}

The divisibility theory of commutative rings is a fundamental and persisting topic in mathematics that entails two main aspects: determining irreducibility and finding a factorization of the reducible elements in the ring. Prominent examples are the ring of integers $\mathbb{Z}$ and the ring of polynomials $\mathbb{Z}[x]$. It is then natural to investigate the arithmetic properties of $\mathbb{Z}[[x]]$, the ring of formal power series with integer coefficients. While polynomials in $\mathbb{Z}[x]$ can be seen as power series over the integers, the factorization properties over $\mathbb{Z}[x]$ and over $\mathbb{Z}[[x]]$ are in general unrelated; cf. [3]. In [4], the authors studied this factorization problem exhaustively. In particular, for a class of polynomials parametrized by a prime $p$, a connection between reducibility in $\mathbb{Z}[[x]]$ and the existence of a $p$-adic root with positive valuation was established. Whereas this connection can be certainly explained in structural terms, the role of the root in the factorization process is not obvious.

Motivated by this factorization problem the main goal of this paper is to give a factorization in $\mathbb{Z}[[x]]$ of certain reducible polynomials whose constant term is of the form $p^{w}$ with $p$ prime and $w>1$. Our formulas are given in terms of partial Bell polynomials and rely on the inversion formula of Lagrange.

For the reader's convenience, a short appendix with some of the basic properties and identities for the partial Bell polynomials is included. We finish by observing that most of the results presented here may be applied to polynomials and power series over other commutative rings.

A full version of this extended abstract, including a discussion on Hensel lifts of polynomial roots modulo $p$, has been submitted elsewhere. A preprint is available via the math arxiv, cf. [6]. 


\section{Factorization of polynomials over $\mathbb{Z}[[x]]$}

Let $f(x)=f_{0}+f_{1} x+f_{2} x^{2}+\ldots$ be a formal power series in $\mathbb{Z}[[x]]$. It is easy to prove that $f(x)$ is invertible in $\mathbb{Z}[[x]]$ if and only if $\left|f_{0}\right|=1$. A natural question, initially discussed in [3], is whether or not a non-invertible element of $\mathbb{Z}[[x]]$ can be factored over $\mathbb{Z}[[x]]$. In recent years, this question has been investigated by several authors, leading to sufficient and in some cases necessary reducibility criteria, see e.g. [2, 4, 9]. In particular, [9] deals with the factorization of formal power series over principal ideal domains.

For the case at hand, the following elementary results are known. The formal power series $f(x)=$ $f_{0}+f_{1} x+f_{2} x^{2}+\ldots$ is irreducible in $\mathbb{Z}[[x]]$ if $\left|f_{0}\right|$ is prime, or if $\left|f_{0}\right|=p^{w}$ with $p$ prime, $w \in \mathbb{N}$, and $\operatorname{gcd}\left(p, f_{1}\right)=1$.

On the other hand, if $f_{0}$ is neither a unit nor a prime power, then $f(x)$ is reducible. In this case, the factorization algorithm is simple and relies on a recursion and a single diophantine equation, see [3, Prop. 3.4].

Finally, in the remaining case when $f_{0}$ is a prime power and $f_{1}$ is divisible by $p$, the reducibility of $f(x)$ in $\mathbb{Z}[[x]]$ is linked to the existence of a $p$-adic root of positive valuation. The goal of this paper is to give an explicit factorization over $\mathbb{Z}[[x]]$ for reducible polynomials of the form

$$
f(x)=p^{w}+p^{m} \gamma_{1} x+\gamma_{2} x^{2}+\cdots+\gamma_{d} x^{d}, \quad m \geq 1, w \geq 2, d \geq 2,
$$

where $\gamma_{1}, \ldots, \gamma_{d} \in \mathbb{Z}$ and $\operatorname{gcd}\left(p, \gamma_{1}\right)=1$. This is the only type of polynomial for which the reducibility and factorization over $\mathbb{Z}[[x]]$ is not straightforward.

Let $\mathbb{Z}_{p}$ denote the ring of $p$-adic integers.

Theorem 2.2 Let $p$ be an odd prime and let $f(x)$ be a polynomial of the form 2.1]. Assume that $f$ has a simple root $r \in p \mathbb{Z}_{p}$ with $v_{p}(r)=\ell \leq m$ and $r=p^{\ell}\left(1+\sum_{j=1}^{\infty} e_{j} p^{\ell j}\right)$ with $e_{j} \in \mathbb{Z}$. Then $f(x)$ admits the factorization

$$
f(x)=\left(p^{\ell}-x-x \sum_{n=1}^{\infty} a_{n} x^{n}\right)\left(p^{w-\ell}+\left(p^{w-2 \ell}+p^{m-\ell} \gamma_{1}\right) x+x \sum_{n=1}^{\infty} b_{n} x^{n}\right),
$$

where the coefficients $a_{n}$ are given by 2.5, and $b_{n}=\hat{b}_{n} / p^{l n}$ with $\hat{b}_{n}$ as in 2.12).

Remark 1 (i) As shown in Lemma 2.13 $\hat{b}_{n}$ is divisible by $p^{\ell n}$, so $b_{n} \in \mathbb{Z}$ for every $n$.

(ii) If $r \in p \mathbb{Z}_{p}$ is a root of $f$ with $v_{p}(r)=\ell \leq m$, then $2 \ell \leq w$.

(iii) If $w \leq 2 m$ and $f$ has a root $r \in p \mathbb{Z}_{p}$, then $v_{p}(r)=\ell \leq m$ holds. If $w>2 m$, then 0 lifts to a $p$-adic root of $f$, but it is not necessarily true that $f$ has a root of valuation less than or equal to $m$. This property depends on the coefficients $\gamma_{2}, \gamma_{3}, \ldots$ However, even if that condition fails, $f(x)$ is still reducible and a factorization can be obtained through the algorithm given in [4] Prop. 2.4].

(iv) A p-adic integer $r$ with $v_{p}(r)=\ell$ can always be written as $r=p^{\ell}\left(e_{0}+\sum_{j=1}^{\infty} e_{j} p^{\ell j}\right)$ with $e_{0} \in \mathbb{Z}_{p}^{*}$. For factorization purposes, we can assume without loss of generality $e_{0}=1$. Otherwise, consider $g(x)=f\left(x / e_{0}^{*}\right)$, where $e_{0}^{*}$ is such that $e_{0} e_{0}^{*}=1\left(\bmod p^{\ell}\right)$.

$(v)$ As discussed in [4], the existence of a root in $p \mathbb{Z}_{p}$ is in many cases (e.g. when $d \leq 3$ ) a necessary condition for the polynomial (2.1) to factor over $\mathbb{Z}[[x]]$. 
Remark 2 If $f$ has a multiple root in $p \mathbb{Z}_{p}$, then $f(x)$ admits the simpler factorization

$$
f(x)=G(x) f_{\text {red }}(x)
$$

where $G(x)=\operatorname{gcd}\left(f(x), f^{\prime}(x)\right) \in \mathbb{Z}[x]$ and $f_{\text {red }}(x)=f(x) / G(x)$.

The proof of the above theorem relies on the following consequence of the inversion formula of Lagrange for formal power series. For a detailed proof and other applications, we refer the reader to [8, Section 3.8] or [7] Section 11.6]. In what follows, $B_{n, j}\left(x_{1}, x_{2}, \ldots\right)$ denotes the $(n, j)$-th partial Bell polynomial, see the appendix.

Lemma 2.3 (cf. [7, Corollary 11.3]) If $\phi(t)$ is a power series of the form

$$
\phi(t)=t\left(1+\sum_{r=1}^{\infty} \alpha_{r} \frac{t^{r}}{r !}\right)
$$

then its formal inverse is given by

$$
\phi^{-1}(u)=u\left(1+\sum_{n=1}^{\infty} \beta_{n} \frac{u^{n}}{n !}\right)
$$

where

$$
\beta_{n}=\sum_{j=1}^{n}(-1)^{j} \frac{(n+j) !}{(n+1) !} B_{n, j}\left(\alpha_{1}, \alpha_{2}, \ldots\right) .
$$

\section{Proof of Theorem 2.2}

Let $r=p^{\ell}\left(1+\sum_{j=1}^{\infty} e_{j} p^{\ell j}\right)$ be the $p$-adic root of $f$ and define

$$
\phi(x)=x E(x) \quad \text { with } \quad E(x)=1+\sum_{j=1}^{\infty} e_{j} x^{j} .
$$

Thus $r=\phi\left(p^{\ell}\right)$ in $\mathbb{Z}_{p}$ and therefore $p^{\ell}=\phi^{-1}(r)$. Define $A(x)=p^{\ell}-\phi^{-1}(x)$. So $A(r)=0$ in $\mathbb{Z}_{p}$, and by Lemma 2.3 , we have

$$
A(x)=p^{\ell}-\phi^{-1}(x)=p^{\ell}-x\left(1+\sum_{n=1}^{\infty} a_{n} x^{n}\right)
$$

where

$$
a_{n}=\frac{1}{n !} \sum_{k=1}^{n}(-1)^{k} \frac{(n+k) !}{(n+1) !} B_{n, k}\left(1 ! e_{1}, 2 ! e_{2}, \ldots\right) \in \mathbb{Z} .
$$

Our goal is to find $B(x) \in \mathbb{Z}[[x]]$ such that $f(x)=A(x) B(x)$. For convenience, consider

$$
\hat{f}(x)=p^{-2 \ell} f\left(p^{\ell} x\right) \text { and } \hat{A}(x)=p^{-\ell} A\left(p^{\ell} x\right) .
$$

Thus

$$
\hat{A}(x)=1-x-x \sum_{n=1}^{\infty} p^{\ell n} a_{n} x^{n} .
$$


Proposition 2.6 The reciprocal of $\hat{A}(x)$ is a power series in $\mathbb{Z}[[x]]$ of the form

$$
\hat{A}(x)^{-1}=\frac{1}{\hat{A}(x)}=1+x+x \sum_{n=1}^{\infty} t_{n} x^{n}
$$

with

$$
t_{n}=1+\sum_{k=1}^{n} p^{\ell k} \frac{n+1-k}{k !} \sum_{j=1}^{k}(-1)^{j} \frac{(n+j) !}{(n+1) !} B_{k, j}\left(1 ! e_{1}, 2 ! e_{2}, \ldots\right) \in \mathbb{Z}
$$

Proof: As an application of Faà di Bruno's formula (cf. Theorem B in [8, Section 3.5]), and using basic properties of partial Bell polynomials, we have

$$
\begin{aligned}
\hat{A}(x)^{-1} & =1+x+\sum_{n=2}^{\infty} \sum_{k=1}^{n} \frac{k !}{n !} B_{n, k}\left(1,2 ! a_{1} p^{\ell}, 3 ! a_{2} p^{2 \ell}, \ldots\right) x^{n} \\
& =1+x+\sum_{n=2}^{\infty} \sum_{k=1}^{n} k !\left(\sum_{j=0}^{k} \frac{n !}{(n-k) ! j !} B_{n-k, k-j}\left(1 ! p^{\ell} a_{1}, 2 ! p^{2 \ell} a_{2}, \ldots\right)\right) \frac{x^{n}}{n !} \\
& =1+x+\sum_{n=1}^{\infty}\left(1+\sum_{k=1}^{n}\left(\sum_{j=1}^{n+1-k} \frac{(n+1-k) !}{k !(n+1-k-j) !} p^{\ell k} B_{k, j}\left(1 ! a_{1}, 2 ! a_{2}, \ldots\right)\right)\right) x^{n+1} \\
& =1+x+x \sum_{n=1}^{\infty}\left(1+\sum_{k=1}^{n} \frac{p^{\ell k}}{k !}\left(\sum_{j=1}^{k} \frac{(n+1-k) !}{(n+1-k-j) !} B_{k, j}\left(1 ! a_{1}, 2 ! a_{2}, \ldots\right)\right)\right) x^{n} .
\end{aligned}
$$

In the last step, we declare the interior sum to be zero if $j>\min (k, n+1-k)$. Thus

$$
\begin{aligned}
t_{n} & =1+\sum_{k=1}^{n} \frac{p^{\ell k}}{k !} \sum_{j=1}^{k} \frac{(n+1-k) !}{(n+1-k-j) !} B_{k, j}\left(1 ! a_{1}, 2 ! a_{2}, \ldots\right) \\
& =1+\sum_{k=1}^{n} p^{\ell k} \frac{n+1-k}{k !} \sum_{j=1}^{k}\left(\begin{array}{c}
n-k \\
j-1
\end{array}\right)(j-1) ! B_{k, j}\left(1 ! a_{1}, 2 ! a_{2}, \ldots\right)
\end{aligned}
$$

Now, if we write $k ! a_{k}$ as

$$
k ! a_{k}=\sum_{j=1}^{k}\left(\begin{array}{c}
k+j \\
j-1
\end{array}\right)(j-1) ! B_{k, j}\left(-1 ! e_{1},-2 ! e_{2}, \ldots\right),
$$


then by means of Theorem 15 in [5] we get

$$
\begin{aligned}
\sum_{j=1}^{k}\left(\begin{array}{c}
n-k \\
j-1
\end{array}\right)(j-1) ! & B_{k, j}\left(1 ! a_{1}, 2 ! a_{2}, \ldots\right) \\
& =\sum_{j=1}^{k}\left(\begin{array}{c}
n+j \\
j-1
\end{array}\right)(j-1) ! B_{k, j}\left(-1 ! e_{1},-2 ! e_{2}, \ldots\right) \\
& =\sum_{j=1}^{k}(-1)^{j} \frac{(n+j) !}{(n+1) !} B_{k, j}\left(1 ! e_{1}, 2 ! e_{2}, \ldots\right)
\end{aligned}
$$

In other words, $t_{n}$ has the form claimed in 2.7.

Now, motivated by [2.7), for $n \geq 1$ we consider

$$
T_{n}(x)=1+\sum_{k=1}^{\infty} \frac{n+1-k}{k !}\left(\sum_{j=1}^{k}(-1)^{j} \frac{(n+j) !}{(n+1) !} B_{k, j}\left(1 ! e_{1}, 2 ! e_{2}, \ldots\right)\right) x^{k} .
$$

Lemma 2.8 With $E(x)$ as in 2.4, we have

$$
T_{n}(x)=E(x)^{-n-2}\left(E(x)+x E^{\prime}(x)\right) .
$$

Proof: Fix $n \geq 1$ and denote

$$
\tau_{k}=\frac{1}{k !} \sum_{j=1}^{k}(-1)^{j} \frac{(n+j) !}{n !} B_{k, j}\left(1 ! e_{1}, 2 ! e_{2}, \ldots\right) .
$$

Then

$$
T_{n}(x)=1+\sum_{k=1}^{\infty}\left(1-\frac{k}{n+1}\right) \tau_{k} x^{k}=1+\sum_{k=1}^{\infty} \tau_{k} x^{k}-\frac{1}{n+1} \sum_{k=1}^{\infty} k \tau_{k} x^{k} .
$$

Using again Theorem B in [8, Section 3.5], it follows that $1+\sum_{k=1}^{\infty} \tau_{k} x^{k}=E(x)^{-n-1}$. Therefore,

$$
\begin{aligned}
T_{n}(x) & =E(x)^{-n-1}-\frac{1}{n+1} x \frac{d}{d x}\left(E(x)^{-n-1}\right) \\
& =E(x)^{-n-1}+x E(x)^{-n-2} E^{\prime}(x)=E(x)^{-n-2}\left(E(x)+x E^{\prime}(x)\right) .
\end{aligned}
$$

As a direct consequence of this lemma we get the recurrence relation

$$
T_{n-1}(x)=E(x) T_{n}(x),
$$

which can be used to define $T_{0}(x)$ and $T_{-n}(x)$ for $n \geq 1$. More precisely, we let

$$
T_{0}(x)=E(x) T_{1}(x) \text { and } T_{-n}(x)=E(x)^{n+1} T_{1}(x) \text { for } n \geq 1 .
$$


Given that

$$
\hat{f}(x)=p^{-2 \ell} f\left(p^{\ell} x\right)=p^{w-2 \ell}+p^{m-\ell} \gamma_{1} x+\sum_{n=2}^{d} p^{\ell(n-2)} \gamma_{n} x^{n},
$$

the relation $T_{n-j}(x)=E(x)^{j} T_{n}(x)$ gives

$$
p^{w-2 \ell} T_{n}(x)+p^{m-\ell} \gamma_{1} T_{n-1}(x)+\sum_{j=2}^{d} p^{\ell(j-2)} \gamma_{j} T_{n-j}(x)=\hat{f}(E(x)) T_{n}(x) .
$$

Moreover, since $E(x)$ is a unit in $\mathbb{Z}[[x]]$, for every $\nu \in \mathbb{Z}$ the function $T_{\nu}(x)$ is in $\mathbb{Z}[[x]]$ and so $T_{\nu}\left(p^{\ell}\right) \in$ $\mathbb{Z}_{p}$.

Lemma 2.11 For $\nu \geq-1$, the $p$-adic numbers $T_{\nu}\left(p^{\ell}\right)$ satisfy

$$
T_{\nu}\left(p^{\ell}\right)-t_{\nu} \equiv 0 \quad\left(\bmod p^{\ell(\nu+2)}\right)
$$

with $t_{\nu}$ as in 2.7) for $\nu>0$ and $t_{0}=t_{-1}=1$.

Proof: For $\nu=n \geq 1$ the statement is a consequence of the fact that $t_{n}$ is the $n$-th partial sum of $T_{n}\left(p^{\ell}\right)$ and the coefficient of $x^{n+1}$ in $T_{n}(x)$ is zero. Further, given that

$$
E\left(p^{\ell}\right)=1+p^{\ell} e_{1}+O\left(p^{2 \ell}\right) \text { and } T_{1}\left(p^{\ell}\right)=1-p^{\ell} e_{1}+O\left(p^{2 \ell}\right),
$$

we have

$$
T_{0}\left(p^{\ell}\right)=\left(1+p^{\ell} e_{1}+O\left(p^{2 \ell}\right)\right)\left(1-p^{\ell} e_{1}+O\left(p^{2 \ell}\right)\right) \equiv 1 \quad\left(\bmod p^{2 \ell}\right) .
$$

This implies $T_{0}\left(p^{\ell}\right)-t_{0} \equiv 0\left(\bmod p^{2 \ell}\right)$.

Finally, since $T_{-1}\left(p^{\ell}\right)=E\left(p^{\ell}\right)^{2} T_{1}\left(p^{\ell}\right)$, and because $E\left(p^{\ell}\right)^{2}$ and $T_{1}\left(p^{\ell}\right)$ are both of the form $1+O\left(p^{\ell}\right)$, we get $T_{-1}\left(p^{\ell}\right) \equiv 1\left(\bmod p^{\ell}\right)$, hence $T_{-1}\left(p^{\ell}\right)-t_{-1} \equiv 0\left(\bmod p^{\ell}\right)$.

Using $\hat{f}(x)$ as in 2.9), we now define

$$
\hat{B}(x)=\hat{f}(x) \hat{A}(x)^{-1}=\left(p^{w-2 \ell}+p^{m-\ell} \gamma_{1} x+\sum_{n=2}^{d} p^{\ell(n-2)} \gamma_{n} x^{n}\right)\left(1+x+x \sum_{n=1}^{\infty} t_{n} x^{n}\right)
$$

and write it as

$$
\hat{B}(x)=p^{w-2 \ell}+\left(p^{w-2 \ell}+p^{m-\ell} \gamma_{1}\right) x+x \sum_{n=1}^{\infty} \hat{b}_{n} x^{n}
$$

with

$$
\hat{b}_{n}=p^{w-2 \ell} t_{n}+p^{m-\ell} \gamma_{1} t_{n-1}+\sum_{j=2}^{d} p^{\ell(j-2)} \gamma_{j} t_{n-j} \in \mathbb{Z},
$$

where $t_{n}$ is given by (2.7), $t_{0}=t_{-1}=1$, and $t_{-n}=0$ for $n>1$.

Lemma 2.13 The coefficients $\hat{b}_{n}$ are divisible by $p^{\ell n}$. 
Proof: First of all, since $p^{-\ell} r=E\left(p^{\ell}\right)$ is a $p$-adic root of $\hat{f}$, identity (2.10) implies

$$
p^{w-2 \ell} T_{n}\left(p^{\ell}\right)+p^{m-\ell} \gamma_{1} T_{n-1}\left(p^{\ell}\right)+\sum_{j=2}^{d} p^{\ell(j-2)} \gamma_{j} T_{n-j}\left(p^{\ell}\right)=0 \text { in } \mathbb{Z}_{p} .
$$

Therefore, for $n \geq d-1$,

$$
\begin{aligned}
\hat{b}_{n} & =p^{w-2 \ell} t_{n}+p^{m-\ell} \gamma_{1} t_{n-1}+\sum_{j=2}^{d} p^{\ell(j-2)} \gamma_{j} t_{n-j} \\
& =p^{w-2 \ell}\left(t_{n}-T_{n}\left(p^{\ell}\right)\right)+p^{m-\ell} \gamma_{1}\left(t_{n-1}-T_{n-1}\left(p^{\ell}\right)\right)+\sum_{j=2}^{d} p^{\ell(j-2)} \gamma_{j}\left(t_{n-j}-T_{n-j}\left(p^{\ell}\right)\right),
\end{aligned}
$$

which by Lemma 2.11 is congruent to $0 \bmod p^{\ell n}$. Similarly, for $1 \leq n<d-1$,

$$
\begin{aligned}
\hat{b}_{n} & =p^{w-2 \ell} t_{n}+p^{m-\ell} \gamma_{1} t_{n-1}+\sum_{j=2}^{n+1} p^{\ell(j-2)} \gamma_{j} t_{n-j} \\
& \equiv-\sum_{j=n+2}^{d} p^{\ell(j-2)} \gamma_{j} T_{n-j}\left(p^{\ell}\right) \equiv 0 \quad\left(\bmod p^{\ell n}\right) .
\end{aligned}
$$

Finally, defining $B(x)=p^{\ell} \hat{B}\left(x / p^{\ell}\right)$, we arrive at the factorization $f(x)=A(x) B(x)$.

Remark 3 It is worth mentioning that our method for factorization in $\mathbb{Z}[[x]]$ is not restricted to polynomials and can be applied to power series. As an example, consider

$$
f(x)=9+12 x+7 x^{2}+8 x^{3} \sum_{k=0}^{\infty} x^{k}=9+12 x+7 x^{2}+\frac{8 x^{3}}{1-x},
$$

discussed by Bézivin in [2]. This series is reducible in $\mathbb{Z}[[x]]$ and factors as

$$
f(x)=\frac{(3-x)^{2}(1+x)}{1-x} .
$$

The reader is invited to confirm that the power series version of Theorem 2.2 gives the factorization $f(x)=A(x) B(x)$ with $A(x)=3-x$ and $B(x)=\frac{(3-x)(1+x)}{1-x}$.

An interesting feature of this example is that the partial sums $f_{d}(x)=9+12 x+7 x^{2}+\cdots$ of $f(x)$ of degree $d \geq 2$ are all irreducible in $\mathbb{Z}[[x]]$. This fact was proved in [2] Prop. 8.1], but it can also be derived from Proposition 3.4 of [5] together with the observation that for $d \geq 2$, the polynomial $f_{d}(x)$ has no roots in $p \mathbb{Z}_{p}$. 


\section{Appendix: Some properties of Bell polynomials}

Throughout this paper, we make extensive use of the well-known partial Bell polynomials. For any sequence $x_{1}, x_{2}, \ldots$, the $(n, k)$-th partial Bell polynomial is defined by

$$
B_{n, k}(x)=\sum_{i \in \pi(n, k)} \frac{n !}{i_{1} ! i_{2} ! \cdots}\left(\frac{x_{1}}{1 !}\right)^{i_{1}}\left(\frac{x_{2}}{2 !}\right)^{i_{2}} \ldots
$$

where $\pi(n, k)$ is the set of all sequences $i=\left(i_{1}, i_{2}, \ldots\right)$ of nonnegative integers such that

$$
i_{1}+i_{2}+\cdots=k \text { and } i_{1}+2 i_{2}+3 i_{3}+\cdots=n
$$

Clearly, these polynomials satisfy the homogeneity relation

$$
B_{n, k}\left(a b x_{1}, a b^{2} x_{2}, a b^{3} x_{3}, \ldots\right)=a^{k} b^{n} B_{n, k}\left(x_{1}, x_{2}, x_{3}, \ldots\right) .
$$

Here are other elementary identities (cf. [8, Section 3.3]) needed in this paper:

$$
\begin{gathered}
B_{n, k}\left(\frac{x_{2}}{2}, \frac{x_{3}}{3}, \ldots\right)=\frac{n !}{(n+k) !} B_{n+k, k}\left(0, x_{2}, x_{3}, \ldots\right), \\
B_{n, k}\left(x_{1}+x_{1}^{\prime}, x_{2}+x_{2}^{\prime}, \ldots\right)=\sum_{\substack{\kappa \leq k \\
\nu \leq n}}\left(\begin{array}{l}
n \\
\nu
\end{array}\right) B_{\nu, \kappa}\left(x_{1}, x_{2}, \ldots\right) B_{n-\nu, k-\kappa}\left(x_{1}^{\prime}, x_{2}^{\prime}, \ldots\right), \\
B_{n, k}\left(0, \ldots, 0, x_{j}, 0, \ldots\right)=0, \text { except } B_{j k, k}=\frac{(j k) !}{k !(j !)^{k}} x_{j}^{k} .
\end{gathered}
$$

Also of special interest is the identity

$$
B_{n, k}\left((a)_{1},(a)_{2}, \ldots\right)=\frac{1}{k !} \sum_{j=0}^{k}(-1)^{k-j}\left(\begin{array}{l}
k \\
j
\end{array}\right)(j a)_{n}
$$

where $(a)_{n}=a(a-1) \cdots(a-n+1)$. This is a special case of [10, Example 3.2].

For more on Bell polynomials and their applications, see e.g. [1, 7, 8, 10].

\section{References}

[1] E.T. Bell, Exponential polynomials, Ann. of Math., 35 (1934), pp. 258-277.

[2] J.-P. Bézivin, Factorisation de polynômes, unpublished manuscript (in French), 2008, available at http://jp.bezivin.pagesperso-orange.fr/

[3] D. Birmajer and J.B. Gil, Arithmetic in the ring of formal power series with integer coefficients, Amer. Math. Monthly 115 (2008), no. 6, 541-549.

[4] D. Birmajer, J.B. Gil, and M.D. Weiner, Factoring polynomials in the ring of formal power series over $\mathbb{Z}$, Int. J. Number Theory 8 (2012), no. 7, 1763-1776. 
[5] D. Birmajer, J.B. Gil, and M.D. Weiner, Some convolution identities and an inverse relation involving partial Bell polynomials, Electron. J. Combin. 19 (2012), no. 4, Paper 34, 14 pp.

[6] D. Birmajer, J.B. Gil, and M.D. Weiner, On Hensel's roots and a factorization formula in $\mathbb{Z}[[x]]$, preprint, arXiv:1308.2987 [math.NT], August 2013.

[7] C.A. Charalambides, Enumerative Combinatorics, Chapman and Hall/CRC, Boca Raton, 2002.

[8] L. Comtet, Advanced Combinatorics: The Art of Finite and Infinite Expansions, D. Reidel Publishing Co., Dordrecht, 1974.

[9] J. Elliott, Factoring formal power series over principal ideal domains, preprint, arXiv:1107.4860v4 [math.AC], 2011.

[10] W. Wang and T. Wang, General identities on Bell polynomials, Comput. Math. Appl. 58 (2009), no. $1,104-118$. 
